\title{
Downregulation of miR-214-3p May Contribute to Pathogenesis of Ulcerative Colitis via Targeting STAT6
}

\author{
Jin-an Li, Yong-duo Wang, Kui Wang, Zhen-lan Wang, \\ Dao-yong Jia, Bi-ying Yang, and Chang-bing Xiong \\ Department of Anus \& Intestine Surgery, Three Gorges Central Hospital, Chongqing 404000, China \\ Correspondence should be addressed to Jin-an Li; lijinan2016@126.com
}

Received 21 December 2016; Revised 30 April 2017; Accepted 30 May 2017; Published 2 July 2017

Academic Editor: Xin-yuan Guan

Copyright (c) 2017 Jin-an Li et al. This is an open access article distributed under the Creative Commons Attribution License, which permits unrestricted use, distribution, and reproduction in any medium, provided the original work is properly cited.

\begin{abstract}
MicroRNAs (miRs) are small noncoding RNA molecules and recently have demonstrated that altered expression and functions are their tight association with ulcerative colitis (UC). Previous microarray study reported that miR-214 was downregulated in the sigmoid colon of patients with active UC, but the roles of miR-214 in the pathogenesis of UC remain to be elucidated. In this study, significant lower level of miR-214-3p and higher level of STAT6 in the intestinal mucosa of active UC patients compared with the health controls were confirmed by quantitative real-time PCR. Results of luciferase reporter assays and western blot demonstrated that miR-214-3p directly targets STAT6 and negatively regulates the expression of STAT6 at the posttranscriptional level. Furthermore, the expression of miR-214-3p was decreased in TNF- $\alpha$ treated HT29 cells and STAT6 protein level was increased in a time-dependent manner. Silenced STAT6 and upregulation of miR-214-3p could decrease the level of INF- $\gamma$ in TNF- $\alpha$ treated HT29 cells. Additionally, the results of the present study indicate that miR-214-3p and STAT6 axis may be a novel therapeutic target for intestinal inflammation of patients with active UC.
\end{abstract}

\section{Introduction}

Ulcerative colitis (UC) is a common inflammatory bowel disease (IBD), and bloody purulent stool, recurrent diarrhea, and abdominal pain are the main clinical manifestations [1]. The pathogenesis of UC involves complex interactions of multiple factors, including genetic, infectious, environmental, immunity, and psychological factors. It is remarkable that the intestinal immune system dysregulation and increased secretion of proinflammatory cytokines may be the principal pathogenesis of intestinal inflammation [2]. However, the precise etiology of UC is still unknown.

UC is a complex multifactorial polygenic disease; it develops under the influence of the genetic component and other risk factors. Numerous dysregulated genes can influence the development of the disease via certain signaling pathways [3]. Available data indicated that many studies have focused on expression and functions of miRs in kinds of IBD, especially in UC [4]. miRs are small noncoding RNA oligonucleotides and endogenous and short single stranded molecules that have emerged as key regulators of biological and pathological processes, involved in the pathogenesis of different human inflammatory diseases. Recently, increasing evidences have suggested that miRs play important roles in the regulation of intestinal inflammation in UC. Koukos et al. revealed that reduction of miR-124 appears to increase expression and activity of STAT3 to promote inflammation and pathogenesis of UC in children [5]. MiR-16 was proved to play a regulative role in the immune and inflammatory responses via suppressing the expression of the A2aAR to control the activation of the NF- $\kappa \mathrm{B}$ signaling pathway in ulcerative colitis [6]. Hence, exploring the role of certain miRs in the regulation of intestinal inflammation may be conducive to understanding the pathogenesis of UC. In addition, previous research has shown that the use of serum or plasma miRs proves useful noninvasive tools for the diagnosis of UC [7]. Changes in circulating miRs levels offer the potential for highly sensitive and specific disease detection [8]. Most importantly, circulating miRs could reflect miRNA expression changes in the diseased tissues, and in 
TABLE 1: Sequences for primers.

\begin{tabular}{lcc}
\hline Name & Direction & Primer $\left(5^{\prime}-3^{\prime}\right)$ \\
\hline \multirow{2}{*}{ miR-214-3p } & Forward & ACAGCAGGCACAGACAGG \\
& Reverse & GTGCAGGGTCCGAGGT \\
U6 snRNA & Forward & CTCGCTTCGGCAGCACA \\
& Reverse & AACGCTTCACGAATTTGCGT \\
STAT6 & Forward & CCTCGTCACCAGTTGCTT \\
& Reverse & TCCAGTGCTTTCTGCTCC \\
GAPDH & Forward & GAAGGTGAAGGTCGGAGT \\
& Reverse & GAAGATGGTGATGGGATTTC \\
\hline
\end{tabular}

the literatures two of the UC-associated miRs (miR-21 and miR-155) were identified as also differentially expressed in UC biopsy tissues [9], indicating that miRs represent a potential clinical marker for detection, prognosis, and therapeutic development in patients with UC.

MiR-214-3p is ubiquitously expressed in multiple human tissues and related to quite a few diseases [10-12]. Earlier studies have shown that miR-214 associated with human cancer, including colonic carcinoma, endometrial carcinoma, breast cancer, and gastric cancer $[13,14]$. One previous study briefly mentioned the aberrant expression of miR-214 in UC [15], but the role of miR-214-3p and its target genes in UC were not mentioned. In this study, we characterized that the level of miR-214-3p was significantly decreased and the expression of STAT6 was increased in UC patients compared to health controls. Bioinformatics approaches demonstrated that miR-214-3p targeted the $3^{\prime}$-UTR of STAT6 mRNA to inhibit its translation, which was confirmed by luciferase reporter assay. In condition, we also demonstrated that miR-214-3p played an important role in the regulation of intestinal inflammation. These findings suggested that miR214-3p could provide a theoretical basis for further study on the pathogenesis and new treatment for UC.

\section{Materials and Methods}

2.1. Human Tissues Samples. Colonic mucosa biopsies from the sigmoid colon of 24 patients with active UC and 20 healthy patients undergoing screening colonoscopies were obtained from The Central Hospital of Chongqing Three Gorges (Chongqing, China) between December 2014 and June 2015. Pathological analysis further confirmed the diagnoses of active UC. In particular, this study was approved by the Ethics Committee at the Central Hospital of Chongqing Three Gorges and all procedures were conducted in compliance with approved guidelines of the Ethics Committee.

\subsection{Isolation of Total RNA and Quantitative Real-Time PCR.} Total RNA was extracted using E.Z.N.A. Total RNA Kit I (Omega, USA) or miRVana miRNA Isolation Kit (Ambion). Quantitative real-time PCR was performed with an SYBR Green I real-time PCR kit (GenePharma, Shanghai, China) according to the manufacturer's instructions with a CFX96 Real-Time PCR Detection System (Bio-Rad, Hercules, CA,
USA). Expression of U6 small RNA served as the internal control to normalize the expression level of miR-214-3p. The sequences for primers are reported in Table 1 . The PCR amplification protocol was as follows: an initial $95^{\circ} \mathrm{C}$ for $1 \mathrm{~min}$ and 39 cycles of $95^{\circ} \mathrm{C}$ for $15 \mathrm{~s}, 60^{\circ} \mathrm{C}$ for $30 \mathrm{~s}$, and $72^{\circ} \mathrm{C}$ for $30 \mathrm{~s}$. The experiment was repeated three times, and the experimental data analysis was computed using $2^{-\Delta \Delta C T}$ method.

2.3. Cell Culture. Human intestinal epithelial HT-29 cells were obtained from the Cell Bank of Chinese Academy of Sciences (Shanghai, China) and cultured in RPMI-1640 medium (Hyclone, USA), containing 10\% fetal bovine serum (FBS; Invitrogen, USA), penicillin (100 IU/mL; Sigma, USA), and streptomycin $(100 \mu \mathrm{g} / \mathrm{ml}$, Sigma, USA $)$ in a $5 \% \mathrm{CO}_{2}$ incubator at $37^{\circ} \mathrm{C}$.

2.4. Luciferase Reporter Assay. HT29 cells were seeded in 24well plates and allowed to adhere overnight and cotransfected using Lipofectamine 2000 ${ }^{\mathrm{TM}}$ (Invitrogen, Carlsbad, CA, USA) with wild-type or mutant STAT6 $3^{\prime}$-UTR reporter plasmid and miR-214-3p mimics or control. Luciferase reporter assays were performed 48 hours after transfection using the DualLuciferase Reporter Assay System (Promega Corporation). Firefly luciferase activity was then normalized to Renilla luciferase activity. All experiments were performed in triplicate.

2.5. MiRNA Mimic or Inhibitor Transfection. HT-29 cells were seeded and cultured overnight in 6-well plates till they reached $90 \%$ confluence. The next day, varying amounts of miR-214-3p mimics, miR-214-3p inhibitor, or negative controls were transfected to HT-29 cells using Lipofectamine 2000 (Invitrogen, Carlsbad, CA, USA) according to the manufacturer's instructions. Transfections were repeated at least three times in triplicate. The miR-214-3p mimics, inhibitor, and negative controls were purchased from GenePharma (Shanghai, China).

2.6. RNA Interference. The sequences of small-interfering RNA (siRNA, $5^{\prime}$-AAACGAGAGUGUUAUAACUGUTT-3') used to knock down STAT6 expression was designed and synthesized by GenePharma (Shanghai, China). HT29 cells were transfected with $120 \mathrm{nM}$ STAT6-siRNA or equivalent 


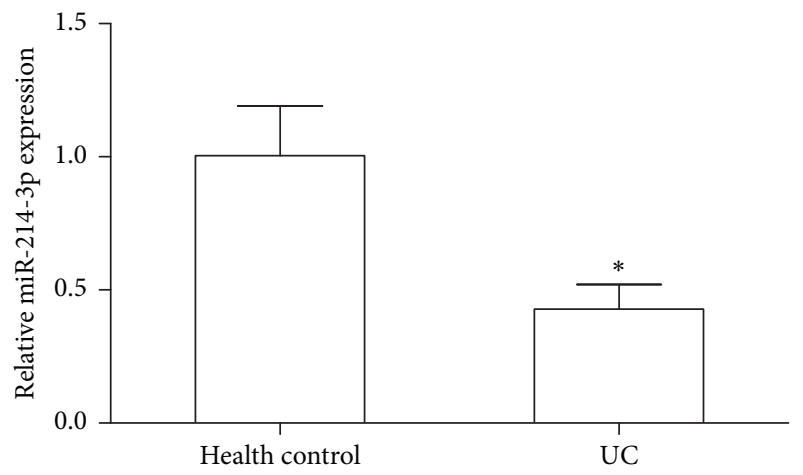

(a)

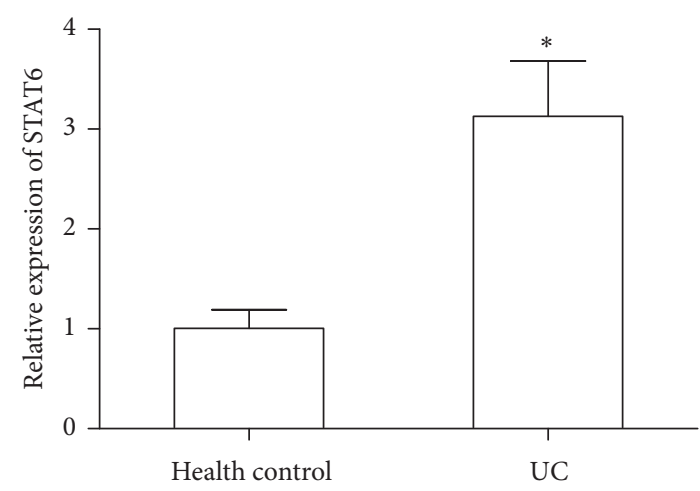

(b)

FIGURE 1: Real-time PCR analyzed the expression levels of miR-214-3p and STAT6 in active UC patients and health controls, respectively. (a) miR-214-3p expression. (b) STAT6 mRNA level. Error bars indicate means \pm SD and, as compared with health controls, ${ }^{*} P<0.05$.

amount of negative control siRNA, using Lipofectamine ${ }^{\mathrm{TM}}$ RNAiMAX transfection reagent (Invitrogen, Carlsbad, CA, USA) as the siRNA transfection reagent according to the manufacturer's protocol.

2.7. Western Blot Analysis. Western blotting analysis was performed as described elsewhere [16]. Total protein from the HT-29 cells was isolated using RIPA Lysis Buffer (Beyotime, China) plus phenylmethanesulfonyl fluoride. Anti-STAT6 primary antibody $(1: 1000$, Abcam, UK) was used in this study. The secondary antibody $(1: 4000$, CST, USA) was goat anti-rabbit IgG horseradish peroxidase (HRP).

2.8. Enzyme-Linked Immunosorbent Assay (ELISA). The cellfree supernatant was collected to evaluate proinflammatory factors after TNF- $\alpha$ treatment and cleared by centrifugation. The concentration of IFN- $\gamma$ was measured by an ELISA kit (R\&D Systems, Minneapolis, MN), according to the manufacturer's protocol.

2.9. Statistical Analysis. The statistical analysis was performed using the SPSS 19.0 software package. All the data values were expressed as mean \pm standard deviation (SD). Statistical difference was determined by one-way ANOVA analysis of variance with multiple comparisons and Student's $t$-test. The results were considered significant at a $P$ value less than 0.05 .

\section{Results}

3.1. Decreased miR-214-3p and Increased STAT6 Are Detected in the Intestinal Mucosa of UC Patients. The expression levels of miR-214-3p and STAT6 were displayed in 24 active UC patients and 20 health controls by quantitative real-time PCR, and the results revealed that the expression of miR-214-3p was significantly downregulated, and significantly high level of STAT6 was observed in the intestinal mucosa of UC patients compared with health controls $(P<0.05$, Figures $1(\mathrm{a})$ and $1(b))$.
3.2. MiR-214-3p Targets the $3^{\prime}$-UTR of STAT6 mRNA. As predicted by TargetScan and PicTar tools analysis, STAT6 was identified as a potential target gene of miR-214-3p and there was complementarity between miR-214-3p and the STAT6 $3^{\prime}$ UTR (Figure 2(a)). A direct interaction between miR-214$3 p$ and STAT6 was assessed by luciferase reporter assay in HT29 cells (Figure 2(b)).

3.3. Endogenous Expression of STAT6 Is Regulated by miR214-3p. Our data showed that alteration of miR-214-3p expression by transfecting miR-214-3p mimics or miR-214$3 p$ inhibitor has changed the STAT6 protein level in HT29 cells. As shown in Figure 3(a), overexpression of miR-214$3 p$ significantly decreased STAT6 expression, while inhibition of miR-214-3p enhanced STAT6 protein level in HT29 cells (Figure 3(b)). These results demonstrate that miR-214-3p can negatively regulate the expression of STAT6 in intestinal epithelial cells.

3.4. TNF- $\alpha$ Regulates miR-214-3p and STAT6 in HT29 Cells. In this study, an in vitro model was established using inflammatory cytokine-stimulated intestinal epithelial cells to further confirm the negative correlation between miR-214-3p and STAT6 expression in active UC tissues. As shown in Figure 4(a), the expression of miR-214-3p decreased with TNF$\alpha(10 \mathrm{ng} / \mathrm{mL})$ stimulation times gradually extending. Instead, the expression of STAT6 increased with TNF- $\alpha(10 \mathrm{ng} / \mathrm{mL})$ stimulation times gradually extending (Figure 4(b)).

3.5. Silencing STAT6 and Overexpressed miR-214-3p Decrease the INF- $\gamma$ Level in TNF- $\alpha$ Treated HT29 Cells. As shown in Figures 5(a) and 5(b), knockdown of the expression of STAT6 protein or upregulated miR-214-3p level significantly decreased the level of INF- $\gamma$ in HT29 cells after 12-hour treatment with TNF- $\alpha(10 \mathrm{ng} / \mathrm{mL})$.

\section{Discussion}

MiRs target mRNAs expression through incompletely or completely binding to the $3^{\prime}$ UTRs of mRNAs and that widely 
Human STAT6 $3^{\prime}$ UTR wild-type
has-miR-214-3p

Human STAT6 3'UTR mutant

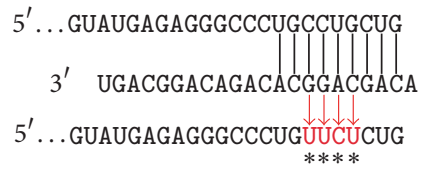

***

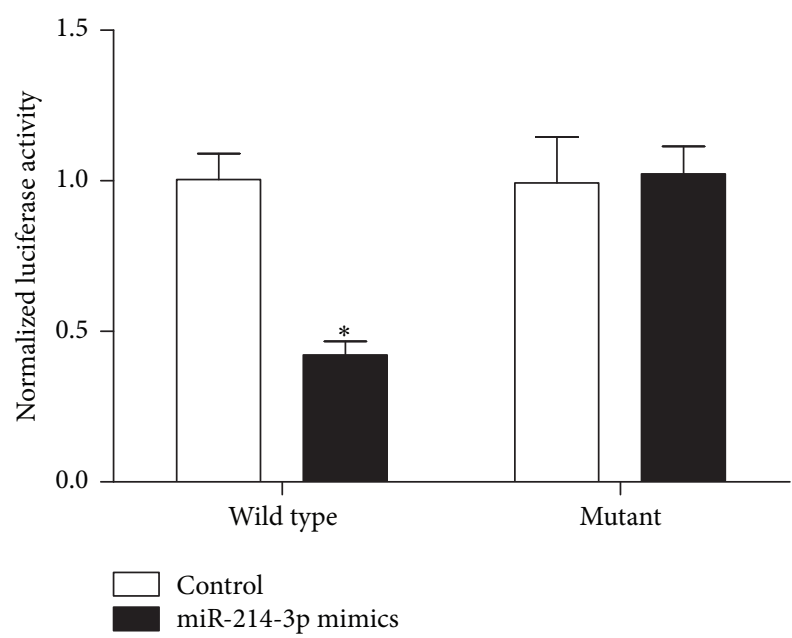

(a)

(b)

Figure 2: MiR-214-3p targets the $3^{\prime}$-UTR of STAT6 mRNA. (a) miR-214-3p/STAT6 alignment by the targets analysis. (b) Relative luciferase activity. Error bars indicate means $\pm \mathrm{SD}$ and, as compared with the control group, ${ }^{*} \mathrm{P}<0.05$.
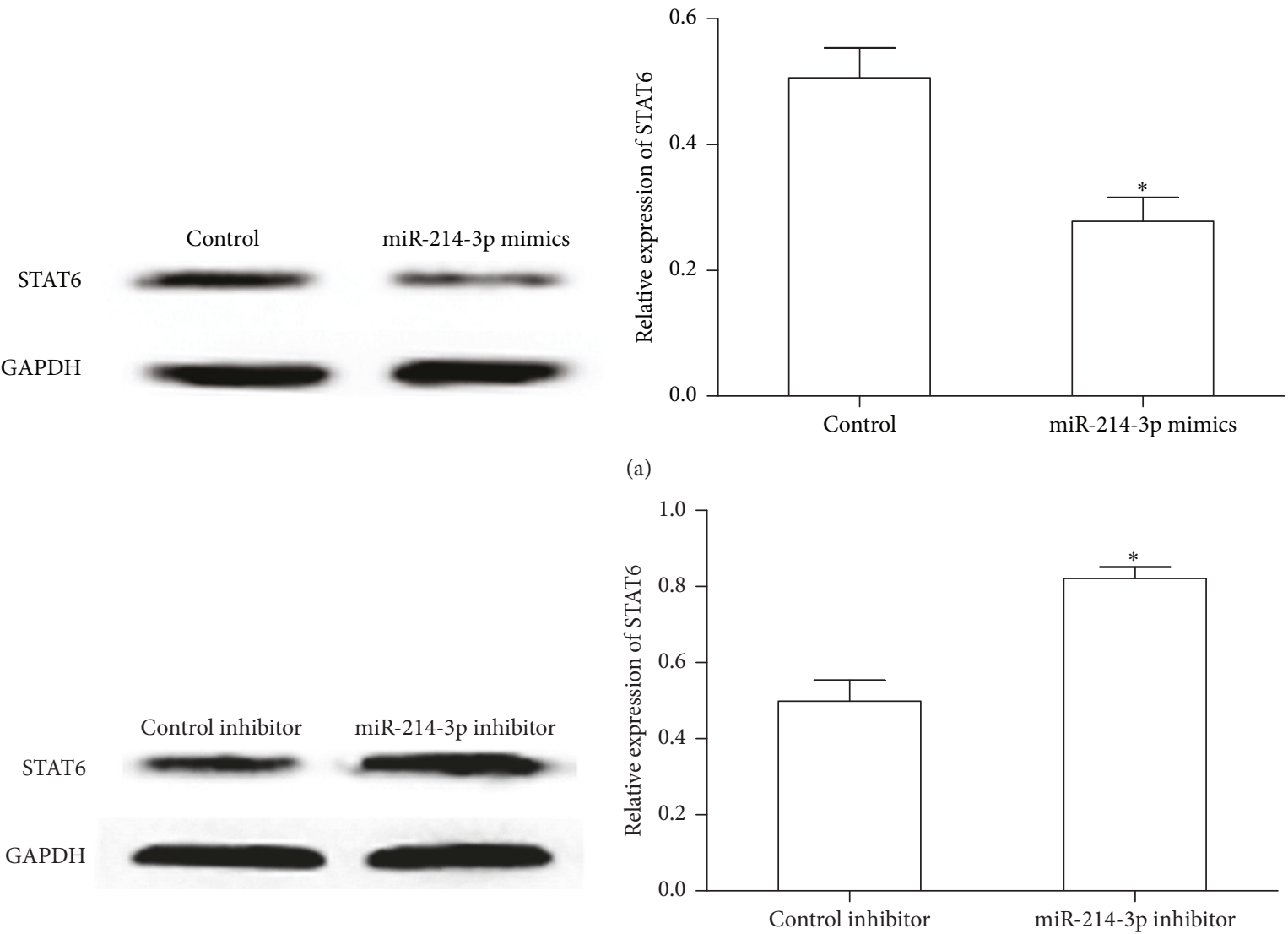

(b)

FIGURE 3: MiR-214-3p regulates the endogenous expression of STAT6. (a) Expression of the STAT6 protein was measured by western blot when HT-29 cells transfected with miR-214-3p mimics. (b) Expression of the STAT6 protein was detected by western blot when HT-29 cells transfected with miR-214-3p inhibitor. Error bars indicate means \pm SD and, as compared with the control group, ${ }^{*} P<0.05$. 


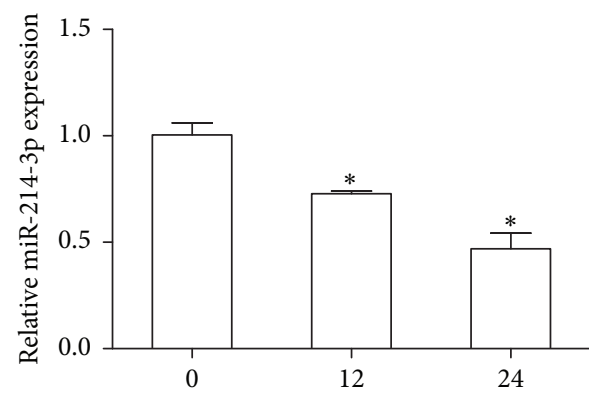

(h)

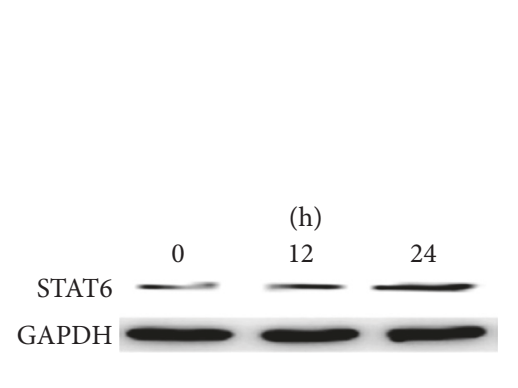

(a)

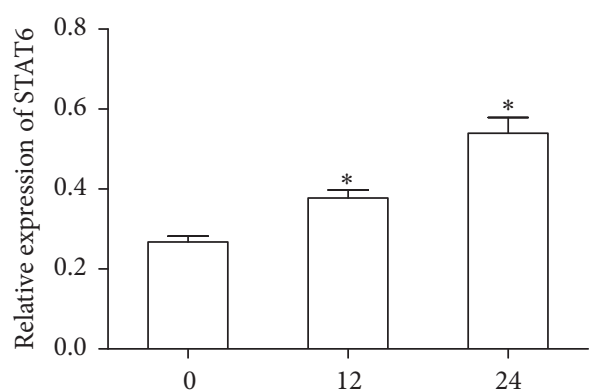

(h)

(b)

FIGURE 4: The effects of miR-214-3p and STAT6 expression after TNF- $\alpha$ treated in HT29 cells. (a, b) miR-214-3p expression and STAT6 protein levels were assessed at different hours after TNF- $\alpha$ treatment by using real-time PCR and western blot, respectively. Error bars indicate means $\pm \mathrm{SD}$ and, as compared with the control group, ${ }^{*} P<0.05$.

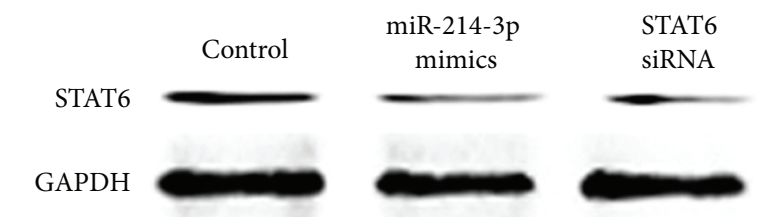

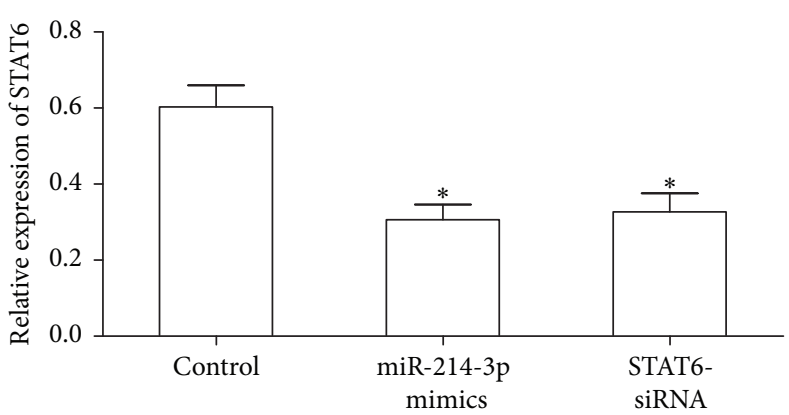

(a)

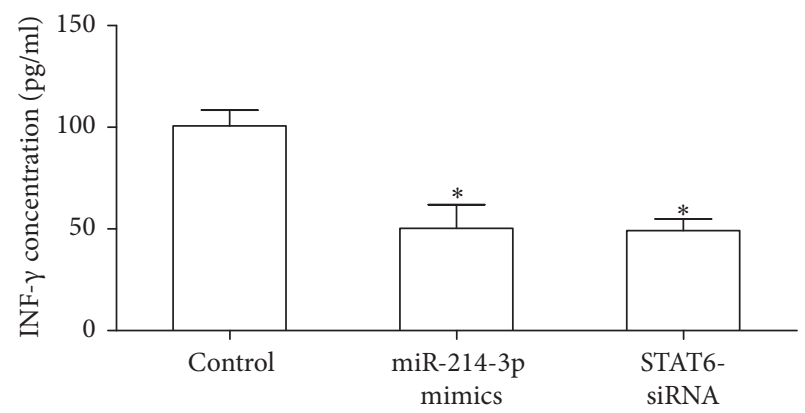

(b)

FIGURE 5: Effect of TNF- $\alpha$ treatment on IFN- $\gamma$ expression when downregulated expression of STAT6 by translating the STAT6-siRNA or miR-214-3p mimics. (a) STAT6 expression after translating the STAT6-siRNA or miR-214-3p mimics in TNF- $\alpha$ treated HT29 cells. (b) INF- $\gamma$ expression was detected by ELISA assay. Error bars indicate means \pm SD and, as compared with the control group, ${ }^{*} P<0.05$.

involves various biological processes, such as inflammation [17], cell proliferation [18], cell differentiation [19], and metabolism [20]. In this study, we confirmed that miR-214-3p is expressed at a low level in the colonic mucosa, suggesting that the abnormal expression of miR-214-3p may be related to the disease activity and the lesion region of UC. For further insight into the function of miR-214-3p, TargetScan and PicTar analyses performed target searches, and the result suggested that STAT6 is a potential target gene of miR-214$3 p$. In addition, we constructed STAT6 $3^{\prime}$ UTR luciferase reporter vector and mutant after transfection of HT29 cells, and results showed that miR-214-3p inhibited the expression of STAT 6 by combining with the $3^{\prime}$ UTR specific sites, and the conclusion was further confirmed by the simulation of miR214-3p mimics and the inhibitor.

Over the past few decades, although the pathogenesis of UC has not elucidated clearly, JAK/STATs mediated signaling pathway in the occurrence of UC is becoming increasingly important to clarify the pathogenesis of UC. The abnormal expression of STATs protein is closely related to the occurrence of many immune abnormalities; especially STAT6 is the key regulator of $\mathrm{T}$ cell development, differentiation, and Th1/Th2 balance [21]. Previous studies have found that upregulation of STAT6 expression in the intestinal mucosa of UC and increased colonic epithelial STAT6 phosphorylation participated in the pathogenesis of UC $[22,23]$. In general, activated STAT6 dimers translocate to the nucleus, bind specific consensus sequences, and promote transcription of inflammatory factors and protein synthesis to give rise to the occurrence of inflammatory reaction in IBD. Studies also show that mesenteric lymph node cells from STAT6 $(-/-)$ mice with colitis exhibited reduced secretion of IL-4, IL-13, and IFN- $\gamma$. It implicates that STAT6 plays important roles in regulating Th2-inducing cytokine production and altering epithelial barrier function in the pathogenesis of colitis [24]. Thus, our finding suggests that miR-214-3p may 
involve the occurrence of UC induced by inflammatory reaction via regulation of STAT6 expression and promoting the production and secretion of proinflammatory cytokines in intestinal epithelial cells.

TNF- $\alpha$ is a kind of cytokine with multiple biological functions, which is an important mediator of immune balance and plays an important role in inflammatory reaction and immune defense [25]. Previous study showed that TNF- $\alpha$ participate in the regulation of the expression of immune regulatory genes and the activation of signal transduction involving the process of inflammatory reaction [26]. In this study, an in vitro model of UC has been successful established by using TNF- $\alpha$ to stimulate HT29 cells to explore the changes of miR-214-3p expression and STAT6 protein level. The results demonstrated that the expression of miR-214$3 p$ decreased gradually with the prolonging of stimulation time, while the expression of STAT6 increased gradually, indicating that miR-214-3p and STAT6 were closely related to the occurrence of inflammation.

UC acts as a chronic persistent excessive inflammatory immune response disease and the excessive inflammatory factors secreted by the lesion involve the occurrence, development, and prognosis of UC, resulting in persistent intestinal inflammation and tissue damage [27]. INF- $\gamma$ is an important proinflammatory cytokines and the amount of INF- $\gamma$ in serum and intestinal mucosa of UC patients increased significantly usually leading to local inflammatory reaction. In this context, miR-214-3p mimics were transfected into TNF$\alpha$ treated HT-29 cells to determine whether the effect of miR214-3p on cytokines secretion was regulated by the expression of STAT6 and results showed enhanced cellular expression of miR-214-3p using miR-214-3p mimics decreased secretion of IFN $-\gamma$ by inhibiting the transcription of STAT6, and similar result was found from transfection with STAT6-siRNA. As expected, our data suggest that miR-214-3p mediated changes in STAT6 seemed to result in changes in proinflammatory cytokine secretion in UC.

Remarkably, it has been well accepted that miRs regulate the expression of hundreds of target genes, and each gene is usually targeted by multiple miRs. Several recent studies have found that combination treatment of two miRs shows synergistically increased anticancer activities. Combination of miR-21 and miR-146a has a greater protective effect against cardiac ischemia/hypoxia-induced apoptosis as compared to these miRs applied individually [28]. Coinhibition of miR$10 \mathrm{~b}$ and miR-21 exerts synergistic inhibition on the proliferation and invasion of human glioma cells [29]. Whether there exists a synergistic action between miR-214-3p and other miRs in the regulation of intestinal inflammation in UC still needs more deliberation.

\section{Conclusions}

This study was focused on the expression of miR-214-3p in UC patients and the function of miR-214-3p in HT29 cells with respect to intestinal inflammation, pushing miRNAbased therapeutics closer to clinical usage. The present study indicates that both miR-214-3p and STAT6 are considered as attractive novel targets for controlling inflammation in UC.

\section{Conflicts of Interest}

The authors declare no conflicts of interest.

\section{References}

[1] C. U. Ghoshal and A. Verma, "Biologicals in treatment of acute ulcerative colitis," Trop Gastroenterol, vol. 36, no. 2, pp. 80-85, 2015.

[2] J. D. Feuerstein and A. S. Cheifetz, "Ulcerative colitis: epidemiology, diagnosis, and management," Mayo Clinic Proceedings, vol. 89, no. 11, pp. 1553-1563, 2014.

[3] W. Zhao, L. Qi, Y. Qin et al., "Functional comparison between genes dysregulated in ulcerative colitis and colorectal carcinoma," PLoS ONE, vol. 8, no. 8, Article ID e71989, 2013.

[4] J. S. Schaefer, T. Attumi, A. R. Opekun et al., "MicroRNA signatures differentiate Crohn's disease from ulcerative colitis," BMC Immunology, vol. 16, no. 1, article 69, p. 5, 2015.

[5] G. Koukos, C. Polytarchou, J. L. Kaplan et al., "MicroRNA124 regulates STAT3 expression and is down-regulated in colon tissues of pediatric patients with ulcerative colitis," Gastroenterology, vol. 145, no. 4, pp. 842-852, 2013.

[6] T. Tian, Y. Zhou, X. Feng et al., "MicroRNA-16 is putatively involved in the NF- $\kappa \mathrm{B}$ pathway regulation in ulcerative colitis through adenosine A2a receptor (A2aAR) mRNA targeting," Scientific Reports, vol. 6, Article ID 30824, 2016.

[7] S. Ben-Shachar, H. Yanai, H. S. Horev et al., "MicroRNAs expression in the ileal pouch of patients with ulcerative colitis is robustly up-regulated and correlates with disease phenotypes," PLoS ONE, vol. 11, no. 8, Article ID e0159956, 2016.

[8] M. Gazouli, "Circulating microRNAs in Inflammatory Bowel Diseases," EXS, vol. 106, pp. 197-214, 2015.

[9] A. Paraskevi, G. Theodoropoulos, I. Papaconstantinou, G. Mantzaris, N. Nikiteas, and M. Gazouli, "Circulating MicroRNA in inflammatory bowel disease," Journal of Crohn's and Colitis, vol. 6, no. 9, pp. 900-904, 2012.

[10] W.-S. Zhu, C.-M. Tang, Z. Xiao et al., "Targeting EZH1 and EZH2 contributes to the suppression of fibrosis-associated genes by miR-214-3p in cardiac myofibroblasts," Oncotarget, vol. 7, no. 48, pp. 78331-78342, 2016.

[11] Y. Zhang, X. Lv, C. Liu et al., "MiR-214-3p attenuates cognition defects via the inhibition of autophagy in SAMP8 mouse model of sporadic Alzheimer's disease," NeuroToxicology, vol. 56, pp. 139-149, 2016.

[12] D. Li, J. Liu, B. Guo et al., "Osteoclast-derived exosomal miR214-3p inhibits osteoblastic bone formation," Nature Communications, vol. 7, p. 10872, 2016.

[13] T. Ueda, S. Volinia, H. Okumura et al., "Relation between microRNA expression and progression and prognosis of gastric cancer: a microRNA expression analysis," The Lancet Oncology, vol. 11, no. 2, pp. 136-146, 2010.

[14] L. M. Long, B. F. He, G. Q. Huang, Y. H. Guo, Y. S. Liu, and J. R. Huo, "microRNA-214 functions as a tumor suppressor in human colon cancer via the suppression of ADP-ribosylation factor-like protein 2," Oncology Letters, vol. 9, no. 2, pp. 645650, 2015.

[15] M. Min, L. Peng, Y. Yang, M. Guo, W. Wang, and G. Sun, "Microrna-155 is involved in the pathogenesis of ulcerative colitis by targeting FOXO3a," Inflammatory Bowel Diseases, vol. 20, no. 4, pp. 652-659, 2014. 
[16] Y. Yang, Y. Ma, C. Shi et al., "Overexpression of miR-21 in patients with ulcerative colitis impairs intestinal epithelial barrier function through targeting the Rho GTPase RhoB," Biochemical \& Biophysical Research Communications, vol. 434, no. 4, pp. 746-752, 2013.

[17] R. P. Singh, I. Massachi, S. Manickavel et al., "The role of miRNA in inflammation and autoimmunity," Autoimmunity Reviews, vol. 12, no. 12, pp. 1160-1165, 2013.

[18] C. Xie, Y. Han, and Y. Liu, "miRNA-124 down-regulates SOX8 expression and suppresses cell proliferation in non-small cell lung cancer," International Journal of Clinical and Experimental Pathology, vol. 7, no. 11, pp. 6534-6542, 2014.

[19] H. L. Fu, D. P. Wu, X. F. Wang et al., "Altered miRNA expression is associated with differentiation, invasion, and metastasis of esophageal squamous cell carcinoma (ESCC) in patients from Huaian, China," Cell Biochemistry and Biophysics, vol. 67, no. 2, pp. 657-668, 2013.

[20] E. Galka, J. L. Thompson, W. J. Zhang, L. S. Poritz, and W. A. Koltun, "Stat6 (null phenotype) human lymphocytes exhibit increased apoptosis," Journal of Surgical Research, vol. 122, no. 1, pp. 14-20, 2004.

[21] A. A. Shah, P. Leidinger, A. Keller et al., "The intestinal factor Tff3 and a miRNA network regulate murine caloric metabolism," RNA Biology, vol. 8, no. 1, pp. 77-81, 2011.

[22] M. J. Rosen, M. R. Frey, M. K. Washington et al., "STAT6 activation in ulcerative colitis: a new target for prevention of IL-13-induced colon epithelial cell dysfunction," Inflammatory Bowel Diseases, vol. 17, no. 11, pp. 2224-2234, 2011.

[23] Y. Li, J. Deuring, M. P. Peppelenbosch, E. J. Kuipers, C. de Haar, and C. J. van der Woude, "STAT1, STAT6 and adenosine $3^{\prime}, 5^{\prime}$-cyclic monophosphate (cAMP) signaling drive SOCS3 expression in inactive ulcerative colitis," Molecular Medicine, vol. 18, pp. 1412-1419, 2012.

[24] M. J. Rosen, R. Chaturvedi, M. K. Washington et al., "STAT6 deficiency ameliorates severity of oxazolone colitis by decreasing expression of Claudin-2 and Th2-inducing cytokines," Journal of Immunology, vol. 190, no. 4, pp. 1849-1858, 2013.

[25] E. Louis, “The immuno-inflammatory reaction in Crohn's disease and ulcerative colitis: characterization, genetics and clinical application: focus on TNF alpha," Acta Gastro-Enterologica Belgica, vol. 64, no. 1, pp. 1-5, 2001.

[26] L. Meng, W. Xu, L. Guo, W. Ning, and X. Zeng, "Paeonol inhibits the proliferation, invasion, and inflammatory reaction induced by TNF- $\alpha$ in vascular smooth muscle cells," Cell Biochemistry and Biophysics, vol. 73, no. 2, pp. 495-503, 2015.

[27] P. Sarlos, E. Kovesdi, and L. Magyari, "Genetic update on inflammatory factors in ulcerative colitis: review of the current literature," World Journal of Gastrointestinal Pathophysiology, vol. 5, no. 3, pp. 304-321, 2014.

[28] W. Huang, S. Tian, P. Hang, C. Sun, J. Guo, and Z. Du, "Combination of microRNA-21 and microRNA-146a attenuates cardiac dysfunction and apoptosis during acute myocardial infarction in mice," Molecular Therapy - Nucleic Acids, vol. 5, p. e296, 2016.

[29] C. G. Dong, W. K. K. Wu, S. Y. Feng, X. J. Wang, J. F. Shao, and J. Qiao, "Co-inhibition of microRNA-10b and microRNA-21 exerts synergistic inhibition on the proliferation and invasion of human glioma cells," International Journal of Oncology, vol. 41, no. 3, pp. 1005-1012, 2012. 


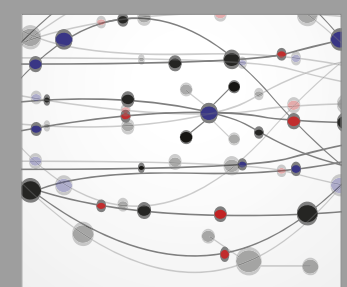

The Scientific World Journal
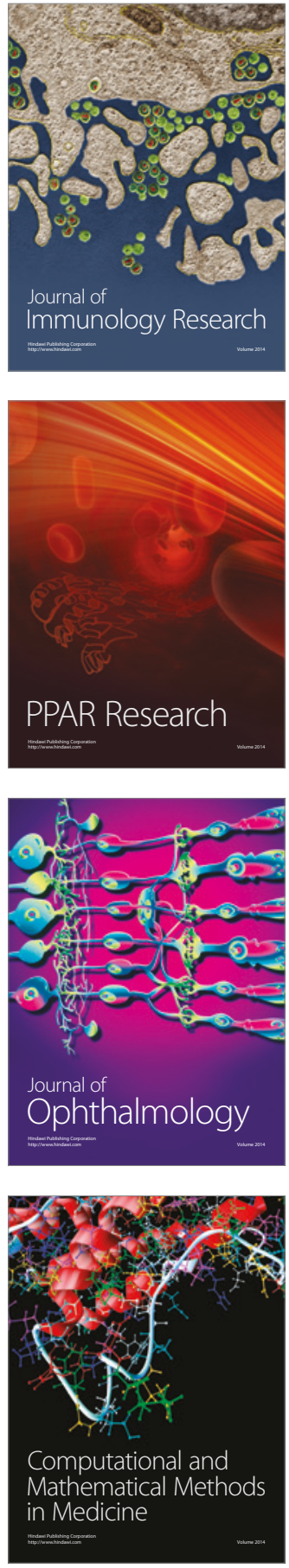

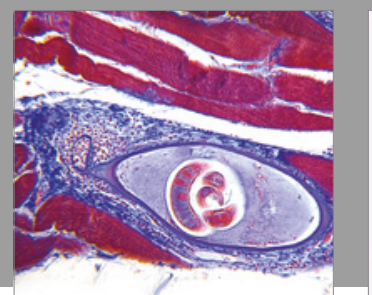

Gastroenterology Research and Practice
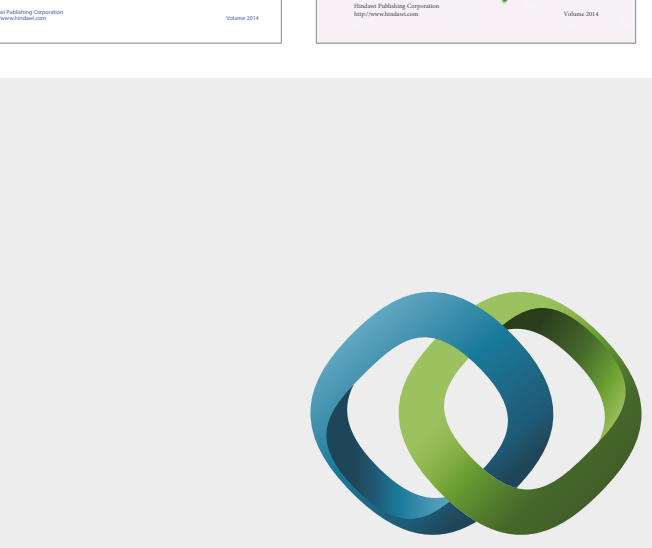

\section{Hindawi}

Submit your manuscripts at

https://www.hindawi.com
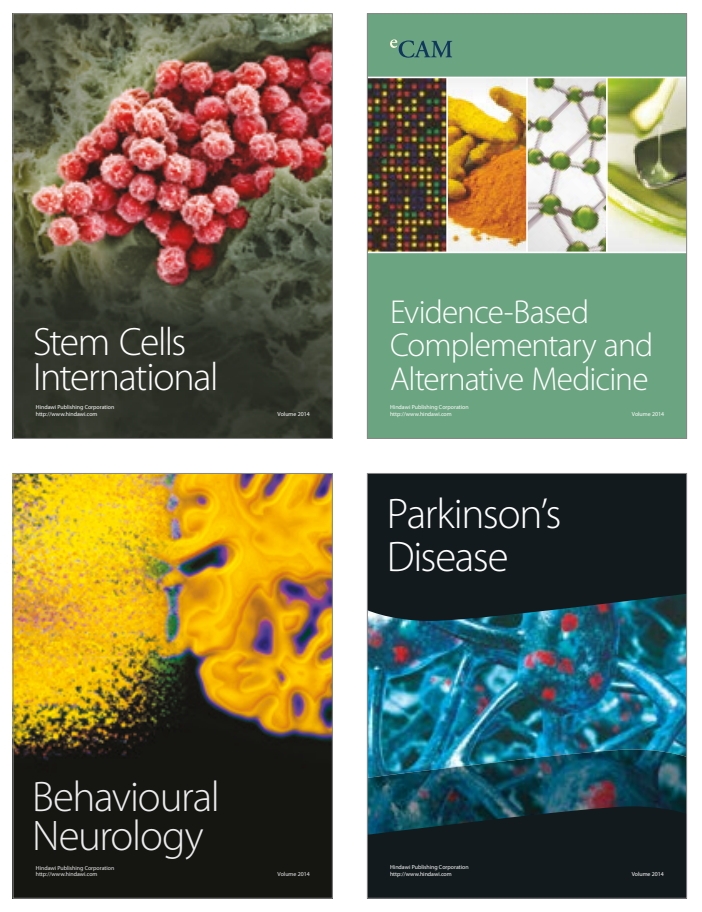
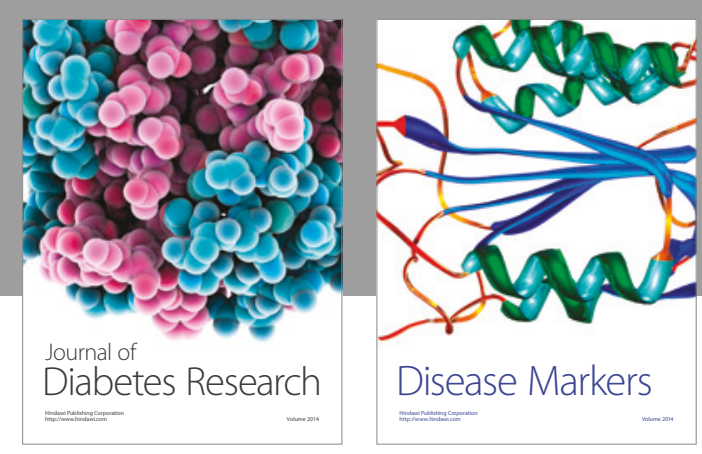

Disease Markers
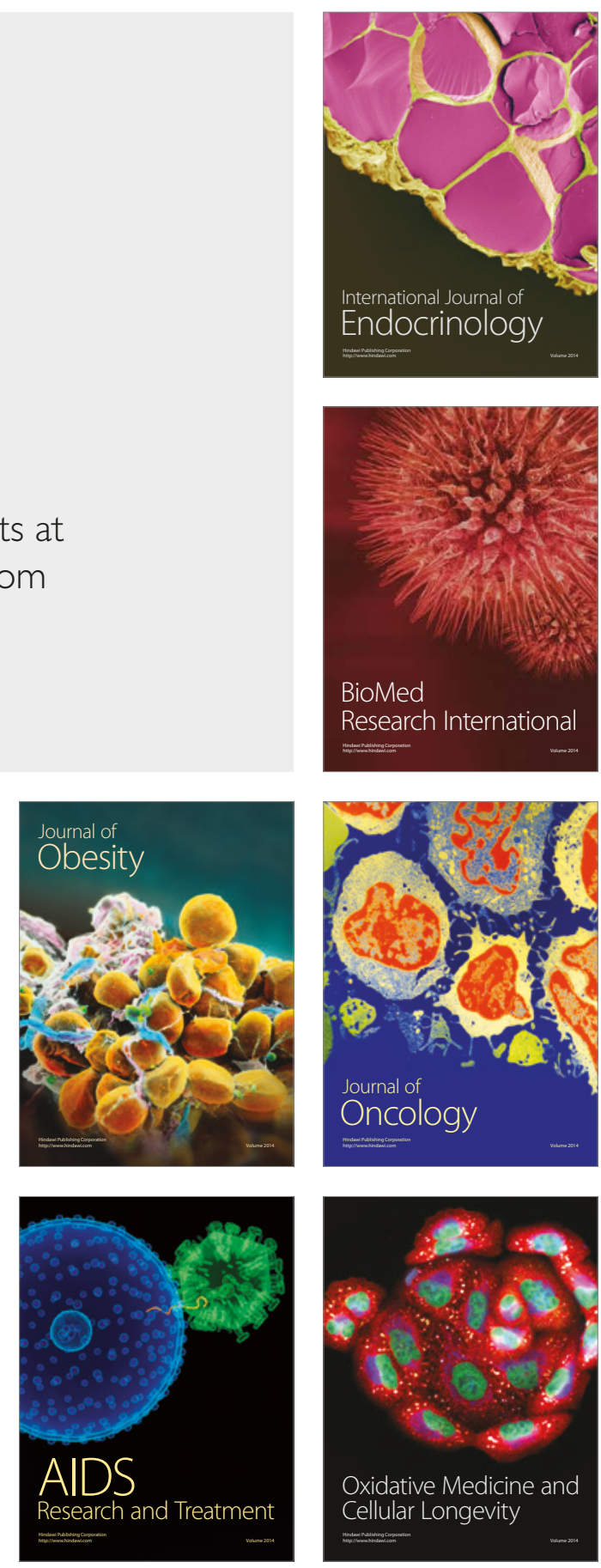